\title{
Undifferentiated embryonal sarcoma of the liver
}

INSERM

\section{Source}

INSERM. (1999). Orphanet: an online rare disease and orphan drug data base.

Undifferentiated embryonal sarcoma of the liver. ORPHA:178315

Embryonal sarcoma of the liver is a rare primary malignant hepatic neoplasm of

childhood of mesenchymal origin. It can rarely occur in adults. It is characterized by

abdominal mass, right upper quadrant or epigastric pain, nausea, anorexia, intermittent fever or headache. 